\title{
Fehler bei der motorischen Neurografie
}

Die motorische Neurografie ist neben der sensiblen Neurografie und der Elektromyografie eine wichtige Methode in der Abklärung von neuromuskulären Erkrankungen. Gut abgeleitete, reproduzierbare Potenziale sind die Voraussetzung für die richtige Befundung und Interpretation im Kontext mit dem klinisch neurologischen Befund. Häufige Fehler kommen sowohl bei der Stimulation als auch bei der Ableitung über innervierten Muskeln und durch fehlenden oder fehlerhaften Einsatz der Erdelektrode vor. Weitere Fehler können bei der Bezeichnung und Messung der Potenziale auftreten. In dem Beitrag werden wichtige Fehler erklärt und die Ursachenbehebung besprochen.

\section{Einleitung}

Die motorische Elektroneurografie ist die Bestimmung und Auswertung der elektrischen AntwortPotenziale stimulierter Muskeln, die durch elektrische Reizung eines peripheren Nervs ausgelöst werden [1].

Die motorische Neurografie ist neben der sensiblen Neurografie und der Elektromyografie eine wichtige Methode in der Abklärung von neuromuskulären Erkrankungen.

Sie wird in der Routine meist durch Medizinisch- technische Assistentinnen oder Assistenten durchgeführt.

Gut abgeleitete, reproduzierbare Potenziale sind die Voraussetzung für die ärztliche Befundung und richtige Interpretation im Kontext mit dem klinisch neurologischen Befund.

In dem Beitrag sollen häufige Fehlerquellen erläutert und die Behebung der Ursachen erklärt werden.

\section{Methode}

Das Prinzip der motorischen Neurografie ist eine supramaximale elektrische Reizung eines motorischen Nervs und die Ableitung eines MuskelsummenaktionsPotenzials (MSAP) über einem von diesem Nerv innervierten Muskel.
Die elektrische Reizung erfolgt mit Oberflächenelektroden, am besten mit Metallelektroden, da diese hygienischer als Filzelektroden sind. Die Kathode (der Minuspol) wird in Richtung des abzuleitenden Muskels gerichtet und über dem Nerv aufgesetzt. Die Stimulationsdauer beträgt 0,2 ms und sollte möglichst nicht überschritten werden. Die Stimulationsintensität wird langsam gesteigert (in 3-5 mA Schritten) bis zur supramaximalen Stimulation. Dies bedeutet, dass 10-20\% über dem Schwellenwert des maximal großen MSAP gereizt wird.

Die Ableitung erfolgt in der Regel ebenfalls mit Oberflächenelektroden, am besten als Klebeelektroden oder es können auch Metallelektroden mit Pflaster fixiert werden. Die Ableitelektrode wird über der Mitte des Muskels (Muskelbauch) geklebt. Die Referenzelektrode wird über einer elektrisch stummen Region, wie dem Sehnenansatz oder einem Knochenvorsprung platziert.

Zur Bestimmung der motorischen Nervenleitgeschwindigkeit (motorisch NLG) ist eine Stimulation des Nerven an 2 unterschiedlichen Punkten erforderlich, da die neuromuskuläre Überleitungszeit an der Endplatte sowie die Erregungsausbreitung im Muskel bei Stimulation des Nerven an einem Ort in die Latenz mit eingeht. Diese Zeiten sind jedoch nicht bekannt bzw. messbar.

Zur Berechnung der motorischen NLG wird die Strecke zwischen beiden Stimulationspunkten (Kathode) durch die Differenz der beiden Latenzen dividiert (motorische NLG = Strecke/Latenzdifferenz).

Die Erdelektrode sollte zwischen Ableitund Reizelektrode positioniert werden.

\section{Häufige Fehler der motorischen Neurografie}

Häufige Fehler bei der motorischen Neurografie kommen sowohl bei der Stimulation als auch bei der Ableitung über innervierten Muskeln vor. Auch durch fehlenden oder fehlerhaften Einsatz der Erdelektrode oder zu geringe Hauttemperatur kann die Ableitung verfälscht werden. Weitere Feh- ler können bei der Bezeichnung und Messung der Potenziale auftreten.

\section{Fehler bei der Stimulation}

Die Stimulation mit ausreichender (supramaximaler), aber nicht zu hoher Intensität an richtiger Stelle ist für eine gute motorische Neurografie sehr wichtig. Folgende Fehler sind möglich:

a) Keine supramaximale Stimulation Die Stimulation muss immer supramaximal erfolgen, da nur dann alle Nervenfasern auch die schnellsten leitenden Nervenfasern erregt und damit auch alle Muskelfasern aktiviert werden. Dies bedeutet, dass 10-20\% über dem Schwellenwert des maximal großen MSAP gereizt wird. Bei nicht supramaximaler Stimulation sind die MSAP und die Latenzen nicht verwertbar (Amplituden kleiner, Latenzen länger, NLG vermindert). Bei tiefer liegenden Nerven ist eine supramaximale Stimulation mit der empfohlenen Reizdauer von 0,2 ms manchmal nicht ausreichend, die Reizdauer kann dann schrittweise bis auf 1,0 ms erhöht werden. Dabei muss auf eine mögliche Mitstimulation benachbarter Nerven geachtet werden. Tiefer liegende Nerven sind z.B. der N.tibialis in der Kniekehle, der N.medianus am Oberarm oder über dem Erb'schen Punkt (supraclavikulär). Durch massive Wassereinlagerungen ins Gewebe an den Extremitäten (Ödeme) können Nerven ebenfalls schlechter stimulierbar sein und es wird eine höhere Stromstärke benötigt.

b) Vertauschen von Kathode und Anode Die Kathode zeigt bei der motorischen Neurografie immer in Richtung des ableitenden Muskels. Werden Kathode und Anode vertauscht dann verlängert sich die Latenz (um 0,2-0,5 ms)

c) Die Kathode wird nicht direkt über dem zu stimulierenden Nerv aufgesetzt, sondern etwas verschoben Dadurch werden höhere Stromstärken zur supramaximalen Reizung benötigt mit daraus resultierender Schmerzhaftigkeit beim Patienten. Daher sollte man die anatomische Lage der Nerven sehr gut kennen und die Kathode nah am Nerv platzieren. Durch nicht korrektes Aufsetzen der Kathode können auch benachbarte Nerven mit stimuliert werden und damit gemischte Potenziale von 
verschiedenen motorischen Nerven aufgezeichnet werden. Zum Beispiel ist eine Mitstimulation des N.ulnaris bei eigentlich gewollter Stimulation des N.medianus am Handgelenk und Ableitung über dem Thenar möglich, da sich am Thenar (Daumenballen) sowohl Medianus- als auch Ulnaris-innervierte Muskeln befinden.

d) $\mathrm{Zu}$ hohe Stimulationsintensität Eine zu hohe Stimulationsintensität kann zu einem großen Stimulationsartefakt führen. Dadurch kann der Abgang des MSAP schlecht abgrenzbar sein. Eine zu hohe Stimulationsintensität kann auch zu einer Mitinnervation benachbarter Nerven und einer Veränderung der MSAP führen.

\section{Fehler bei der Ableitung}

Bei richtiger Position der Ableitelektrode über dem Muskelbauch kommt es zu einem scharf negativen Abgang des MSAP (Definitionsgemäß wird der Abgang nach oben als negativ bezeichnet). Ein nicht negativer $A b$ gang, Veränderungen von Amplitude und Form der MSAP, kann verschiedene Ursachen haben.

Bei initial positivem Abgang des MSAP (also nach unten) können folgende Fehler vorliegen:

a) Falsche Position der Ableitelektrode Die Ableitelektrode ist nicht über dem Muskelbauch und damit nicht über der Endplattenregion platziert. Dies muss durch Umkleben der Ableitelektrode behoben werden bis ein scharf negativer Abgang des MSAP aufgezeichnet wird.

b) Ableit- und Referenzelektrode sind vertauscht Beim Vertauschen von Ableit- und Referenzelektrode ist das MSAP gespiegelt mit initial positivem Abgang. Dies muss durch Korrektur und Tauschen der Elektroden behoben werden.

Bei veränderter Form und Amplitude des MSAP können folgende Fehler/Ursachen zu Grunde liegen:

a) Beide Ableitelektroden liegen zu nah beieinander und damit beide über aktivem Muskelgewebe Die Folge wären zu kleine und deformierte MSAP, da beide Elektroden dann über aktivem Muskelgewebe sitzen und es damit zu einer Auslöschung einzelner Potenzialanteile kommt. Die Referenzelektrode soll jedoch über einer elektrisch nicht aktiven Region (also kein Muskel) platziert werden. Ableitelektroden mit festem Abstand (z. B. Blockelektroden) sind daher für die Ableitung eines MSAP nicht geeignet. Ableit- und Referenzelektrode sollten daher immer variabel platzierbar sein.

b) Simultane Stimulation benachbarter Nerven Das MSAP zeigt mehrere Potenzialanteile (Phasen), ist z. B. doppelgipflig bei der Mitstimulation des N.ulnaris bei Stimulation des N.medianus am Handgelenk und Ableitung über dem Thenar. Die Stimulationsintensität sollte verringert werden und die Kathode direkt auf dem zu stimulierenden Nerven aufgesetzt werden.

c) Keine supramaximale Stimulation Die MSAP sind bei proximaler und distaler Stimulation vergleichbar. Bei nicht supramaximaler Stimulation kann das distale MSAP kleiner sein als das proximale oder auch umgekehrt (proximales MSAP kleiner als distales MSAP).

d) Innervationsanomalien Bei Innervationsanomalien kann das MSAP bei distaler Stimulation von der Amplitude niedriger als bei proximaler Stimulation sein. z. B. Martin-Gruber - Anastomose (Abgabe von Fasern des N.medianus an Fasern des N.ulnaris am Unterarm) bei distaler Stimulation des N.medianus MSAP niedriger als bei proximaler Stimulation [2]. Ein weiteres Beispiel ist das Vorhandensein eines N.peronaeus accessorius, bei distaler Stimulation des N. peroneus ist das MSAP niedriger als bei proximaler Stimulation [3].

\section{Fehler bei der Anwendung der Erdelektrode}

Alle Untersuchungen sollten mit einer $\mathrm{Er}$ delektrode durchgeführt werden, da dadurch Artefakte (z. B. 50- Hz- Artefakte) reduziert werden. Folgende Fehler können auftreten:

a) Die Erdelektrode ist zu locker Die Erdelektrode sollte immer guten Kontakt mit der Haut haben.

b) Erdelektrode aus Stoff ist zu trocken Es sollte immer auf eine gewisse Feuchtigkeit der Erdelektrode geachtet werden. c) Falsche Position der Erdelektrode Bei fehlender oder falscher Position der Erdelektrode kann ein großer Stimulationsartefakt mit Schwierigkeiten der Abgrenzung des Potenzialbeginns auftreten. Die Erdelektrode sollte deshalb immer zwischen Ableit- und Reizelektrode (Kathode) positioniert werden.

d) Kabelbruch Insbesondere bei fehlenden MSAP sollten alle Kabel auf Brüche überprüft werden.

e) Umliegende elektrische Geräte Bei weiter bestehendem 50- Hz-Artefakt andere elektrische Geräte (z. B. Lampe, Handy...) ausschalten.

\section{Fehler beim Setzen der Parameter und bei der Parameterberechnung}

a) Latenz Die Latenz ist die Zeit vom Reizbeginn bis zum Beginn des MSAP, also dem negativen Abgang von der Grundlinie. Darin enthalten sind die Depolarisation des Nerven, die Zeit der Nervenleitung vom Stimulationsort bis zur Endplatte, die neuromuskuläre Übertragungszeit und die Zeit bis zur Muskelerregung. Fehler treten bei der Bestimmung der Latenz bei unterschiedlichen Verstärkungen oder unterschiedlichen Zeitachsen auf. Die Latenz wird mit höherer Verstärkung kürzer gesetzt. Die Latenz verlängert sich, je stärker die Zeitachse auseinandergezogen ist. Daher sollte die Messung der Latenz immer bei gleicher Verstärkung (z.B. 0,2mV/Div) und gleicher Zeitachse (5ms/Div) durchgeführt werden.

b) Distal motorische Latenz (DML) Sie bezeichnet die Zeit bis zum Beginn des MSAP bei distaler Stimulation. Diese ist abhängig von der Strecke zwischen Reiz- und Ableitelektrode und der Temperatur (bei niedrigen Temperaturen, langsamere Leitung). Daher ist für die Messung der distal motorischen Latenz zu beachten, dass sie immer bei gleicher Entfernung (z. B. $7 \mathrm{~cm}$ bei Stimulation des N.medianus am Handgelenk) und warmen Extremitäten erfolgt.

c) Amplitude Die Amplitude wird zwischen Grundlinie und negativer Spitze (negative Amplitude, base -to-peak ) oder von der negativen zur positiven Spitze (peak-to-peak Amplitude) gemessen. Die Amplitude ist nur bei supramaximaler Stimulation zu verwerten und gilt als Maß 


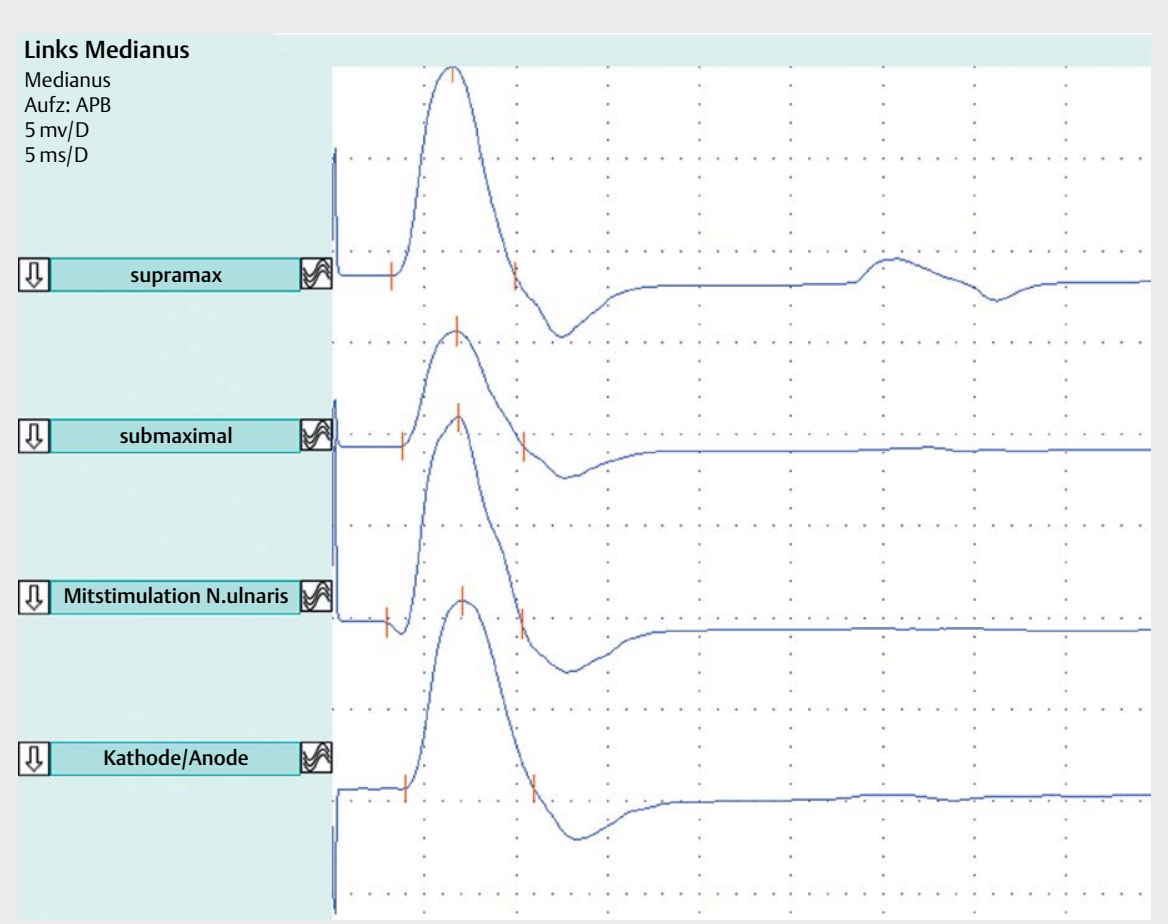

Abb. 1 Ableitung eines MSAP über dem M.abductor poll breves (APB) am Thenar mit unterschiedlicher Stimulation des N.medianus.

1. Spur: normal. supramaximale Stimulation, scharfer negativer Abgang des MSAP, normale distal motorische Latenz (DML)

2. Spur: Fehler, zu geringe Stimulationsintensität. längere DML, niedrige Amplitude des MSAP

3. Spur: Fehler, zu hohe Stimulationsintensität und Kathode ulnar verrutscht. Positiver Abgang durch Mitstimulation des N.ulnaris

4. Spur: Fehler, Kathode und Anode vertauscht. Längere DML

Tab. 1 Stimulationsintensitäten (letzte Spalte) und Messparameter (DML, Amplitude) bei unterschiedlichen Fehlern entsprechend Spur 1 bis Spur 2 in $>$ Abb. 1.

\section{Links Motorisch Medianus}

\begin{tabular}{|l|l|l|l|l|r|}
\hline Gebiete & Lat [ms] & Amp [mV] & Dauer [ms] & Fläche [ms * mV] & Stim [mA] \\
\hline Supramax & 3,22 & 11,4 & 6,7 & 40,3 & 9,4 \\
\hline Submaximal & 3,81 & 6,3 & 6,6 & 22,4 & 5,4 \\
\hline Mitstimulation N.ulnaris & 2,94 & 11,2 & 7,4 & 40,8 & 50,8 \\
\hline Kathode/Anode vertausch & 3,94 & 10,2 & 7,0 & 39,7 & 13,4 \\
\hline
\end{tabular}

für die Zahl der erregten Nerven- und Muskelfasern. Zu beachten ist, dass die Amplituden mit zunehmendem Abstand zwischen Stimulations- und Ableitort kleiner werden (bis zu 20\%). Grund dafür ist eine physiologisch unterschiedliche Leitung verschiedener Nervenfasern (temporale Dispersion) und der damit verbundenen gegenseitigen Auslöschung einiger Potenzialanteile.

d) Motorische NLG Zur Bestimmung der motorischen Nervenleitgeschwindigkeit (motorische NLG) ist eine Stimulation des Nervens an 2 unterschiedlichen Punkten erforderlich. Man berechnet die Geschwindigkeit durch Division der Strecke zwischen 2 Stimulationsorten (Kathodenpunkten) durch die Leitungszeit zwischen beiden Stimulationsorten. Die NLG ist immer die Leitgeschwindigkeit der am schnellsten leitenden Nervenfasern. Die Strecke zwischen 2 Stimulationsorten sollte wegen der Zunahme des Messfehlers bei kurzen Distanzen nicht weniger als
$10 \mathrm{~cm}$ betragen. (z.B. Abstand zweier Stimulationspunkte unterhalb und oberhalb des Sulcus ulnaris bei V.a. Sulcus ulnaris Syndrom). Außerdem sollte während der Längenmessung die Stellung der Extremität wie bei der Stimulation beibehalten werden (z. B. bei Messung über einem Gelenk wie dem Ellenbogengelenk, Stimulation und Messung der Strecke in gleicher Gelenkposition). Bei nicht Beachtung werden fehlerhafte NLG berechnet. 


\section{Temperatur als wichtiger}

\section{Einflussfaktor auf die}

\section{motorische Neurografie}

Die motorische Neurografie sollte bei ausreichend warmen Extremitäten durchgeführt werden (an den Handflächen $>33^{\circ} \mathrm{C}$, am Innenknöchel $30^{\circ} \mathrm{C}$ ). Bei zu kalter Hauttemperatur kommt es zu einer Verlängerung der Latenz und Abnahme der NLG. Dabei wird pro ${ }^{\circ} \mathrm{C}$ Temperaturabnahme mit einer Zunahme der Latenz um 0,2 ms und einer Abnahme der NLG um 1,5-2,5 m/s gerechnet. Da insbesondere die Akren kälter als die proximalen Extremitäten sind und die Nerven im distalen Abschnitt meist oberflächlich verlaufen, ist besonders bei der distalen Stimulation auf ausreichende Temperatur zu achten. Die Hauttemperatur sollte mit einem Thermometer gemessen werden. Ist diese zu niedrig, dann muss die Haut aufgewärmt werden. Dies kann durch Waschung (z. B. der Hände) unter warmem Wasser oder eine Wärmelampe erfolgen.

\section{Beispiele}

In der folgenden Abbildung ( $\triangleright$ Abb. 1) werden Kurven des MSAP bei Ableitung über dem Thenar (M.abd.poll.brev.) bei distaler Stimulation des N.medianus am Handgelenk mit konstanter Strecke von $7 \mathrm{~cm}$ dargestellt. Die erste Kurve zeigt eine regelrechte Ableitung. Bei den folgenden Ablei- tungen (Spur 2-4) sind typische Fehler dargestellt. In $>$ Tab. 1 sind die entsprechenden Stimulationsintensitäten und Messparameter abgebildet.

FAZIT

Häufige Fehler bei der motorischen Neurografie kommen sowohl bei der Stimulation als auch bei der Ableitung über innervierten Muskeln und durch fehlenden oder fehlerhaften Einsatz der Erdelektrode vor. Weitere Fehler können bei Nichtbeachtung der Hauttemperatur und bei der Bezeichnung und Messung der Potenziale auftreten. Im Rahmen der routinemäßigen Messung der motorischen Neurografie sollten mögliche Fehler erkannt und vermieden werden. Eine exakte, fehlerfreie motorische Neurografie ist Voraussetzung für eine richtige Befundung und Interpretation dieser im Kontext mit dem klinisch neurologischen Befund.

\section{Interessenkonflikt}

Die Autorinnen/Autoren geben an, dass kein Interessenkonflikt besteht.

\section{Literatur}

[1] Bischoff C, Dengler R, Hrsg. Thiemes EMG ENG Elektromyografie- Elektronneurografie. 4. Aufl. Thieme; 2018

[2] Hopf HC, Hense W. Anomalien der motorischen Innervation an der Hand. EEG, EMG 1975; 5: 220-224

[3] Neundörfer B, Seiberth R. The Accessory Deep Peroneal Nerve. J Neurol 1975; 209: 125-129

\section{Autorinnen/Autoren}

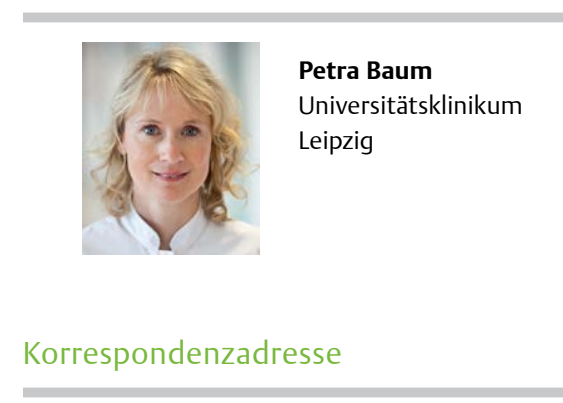

PD Dr. Petra Baum

Universitätsklinikum Leipzig - AöR

Klinik und Poliklinik für Neurologie

Liebigstraße 20

04103 Leipzig

Petra.Baum@medizin.uni-leipzig.de

\section{Bibliografie}

DOI https://doi.org/10.1055/a-1190-9983

Klin Neurophysiol 2020; 51: 174-177

(c) Georg Thieme Verlag KG Stuttgart · New York ISSN 1434-0275 